Stefano Serafin, Mathias W. Rotach, Marco Arpagaus, Ioana Colfescu, Joan Cuxart, Stephan F. J. De Wekker, Mathew Evans,

Vanda Grubišić, Norbert Kalthoff, Thomas Karl, Daniel J. Kirshbaum, Manuela Lehner, Stephen Mobbs, Alexandre Paci, Elisa Palazzi, Adriana Raudzens Bailey, Jürg Schmidli, Georg Wohlfahrt, Dino Zardi

\title{
Multi-scale transport and exchange processes in the atmosphere over mountains
}

\section{Programme and experiment}



Stefano Serafin, Mathias W. Rotach, Marco Arpagaus, Ioana Colfescu, Joan Cuxart, Stephan F. J. De Wekker, Mathew Evans, Vanda Grubišić, Norbert Kalthoff, Thomas Karl, Daniel J. Kirshbaum, Manuela Lehner, Stephen Mobbs, Alexandre Paci, Elisa Palazzi, Adriana Raudzens Bailey, Jürg Schmidli, Georg Wohlfahrt, Dino Zardi

\section{Multi-scale transport and exchange processes in the atmosphere over mountains}

\section{Programme and experiment}

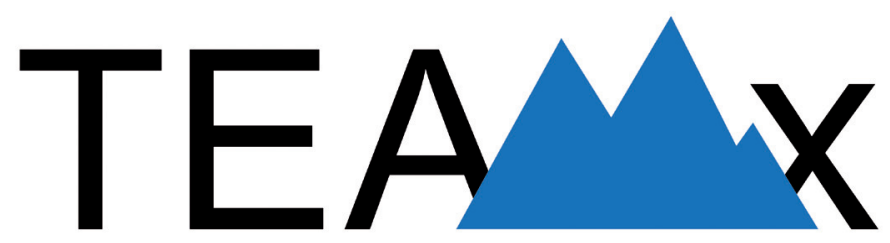


TEAMx Programme Coordination Office

Universität Innsbruck

The TEAMx Programme Coordination Office is financially supported by:

- Center for Climate Systems Modeling

- Karlsruhe Institute of Technology

- Meteo France

- MeteoSwiss

- National Centre for Atmospheric Science

- University of Innsbruck

- University of Trento

- ZAMG

(C) innsbruck university press, 2020

Universität Innsbruck

1. edition

All rights reserved

www.uibk.ac.at/iup

ISBN 978-3-99106-003-1

DOI 10.15203/99106-003-1 


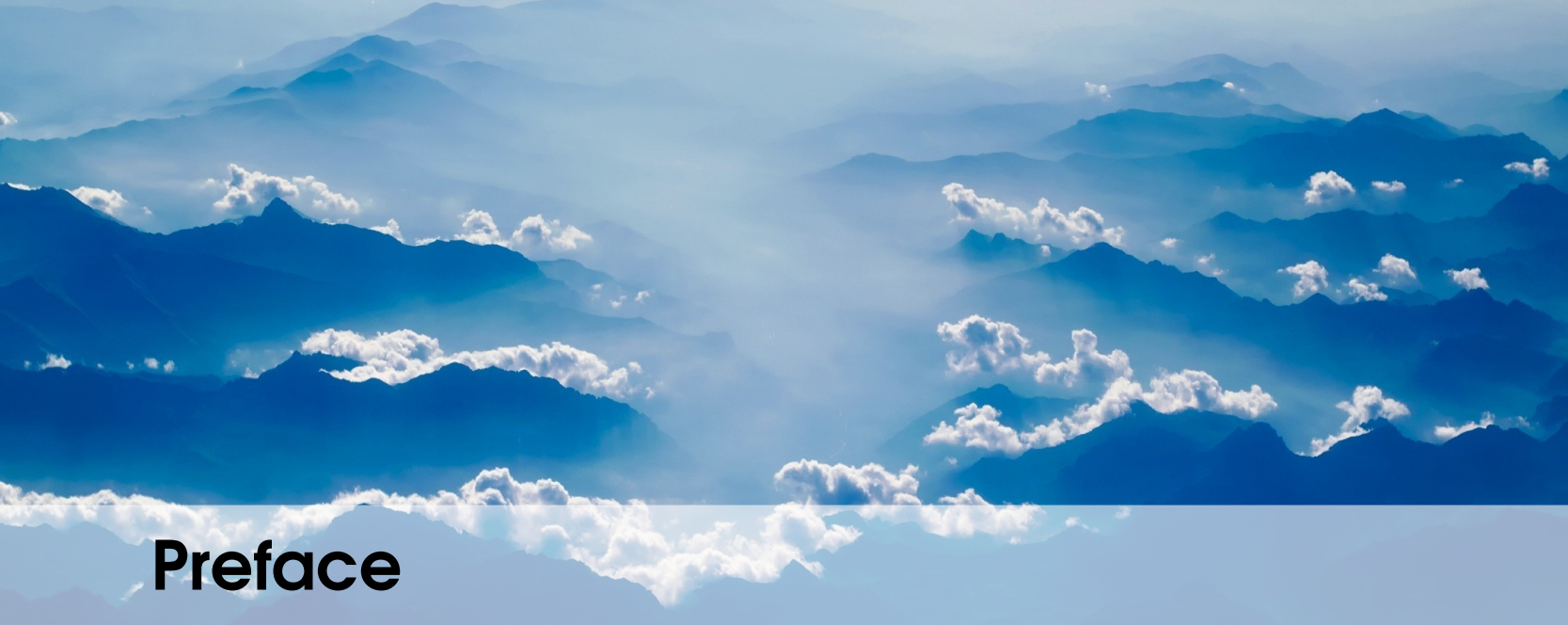

TEAMx is an international research programme that aims at improving the understanding of exchange processes in the atmosphere over mountains at multiple scales and at advancing the parameterizations of these processes in numerical models for weather and climate prediction-hence its acronyms stands for Multi-scale transport and exchange processes in the atmosphere over mountains - Programme and experiment.

TEAMx is a bottom-up initiative promoted by a number of universities, research institutions and operational centres, internationally integrated through a Memorandum of Understanding between interested parties. It is carried out by means of coordinated national, bi-national and multi-national research projects and supported by a Programme Coordination Office at the Department of Atmospheric and Cryospheric Sciences of the University of Innsbruck, Austria.

The present document, compiled by the TEAMx Programme Coordination Office, provides a concise overview of the scientific scope of TEAMx. In the interest of accessibility and readability, the document aims at being self-contained and uses only a minimum of references to scientific literature.

Grey boxes at the beginning of chapters list the literature sources that provide the scientific basis of the document. This largely builds on review articles published by the journal Atmosphere between 2018 and 2019, in a special issue on Atmospheric Processes over Complex Terrain. A few other important literature pieces have been referenced where appropriate. Interested readers are encouraged to examine the large body of literature summarized and referenced in these articles.

Blue boxes have been added to most sub-chapters. Their purpose is to highlight key ideas and proposals for future collaborative research.

\section{Coordinating lead author}

Stefano Serafin (University of Innsbruck)

Contributing authors

Mathias W. Rotach (University of Innsbruck)

Marco Arpagaus (MeteoSwiss)

Ioana Colfescu (Nat. Centre for Atmospheric Science)

Joan Cuxart (University of the Balearic Islands)

Stephan F.J. De Wekker (University of Virginia)

Mathew Evans (Nat. Centre for Atmospheric Science)

Vanda Grubišić (NCAR)

Norbert Kalthoff (Karlsruhe Institute of Technology)

Thomas Karl (University of Innsbruck)

Daniel J. Kirshbaum (McGill University)

Manuela Lehner (University of Innsbruck)

Stephen Mobbs (Nat. Centre for Atmospheric Science)

Alexandre Paci (Meteo France)

Elisa Palazzi (National Research Council of Italy)

Adriana Raudzens Bailey (NCAR)

Jürg Schmidli (Goethe University Frankfurt)

Georg Wohlfahrt (University of Innsbruck)

Dino Zardi (University of Trento) 



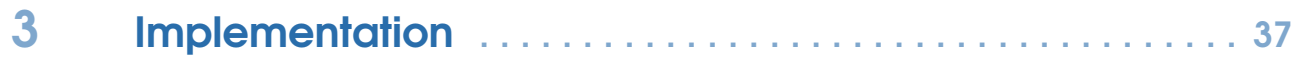

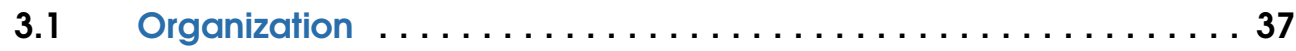

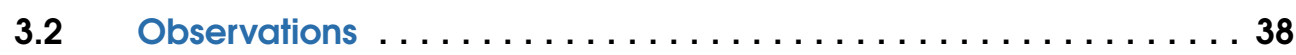

3.3 Numerical experimentation $\ldots \ldots \ldots \ldots \ldots \ldots \ldots \ldots \ldots \ldots$

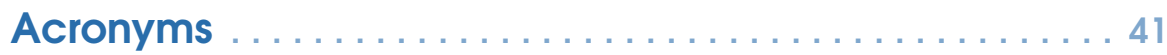




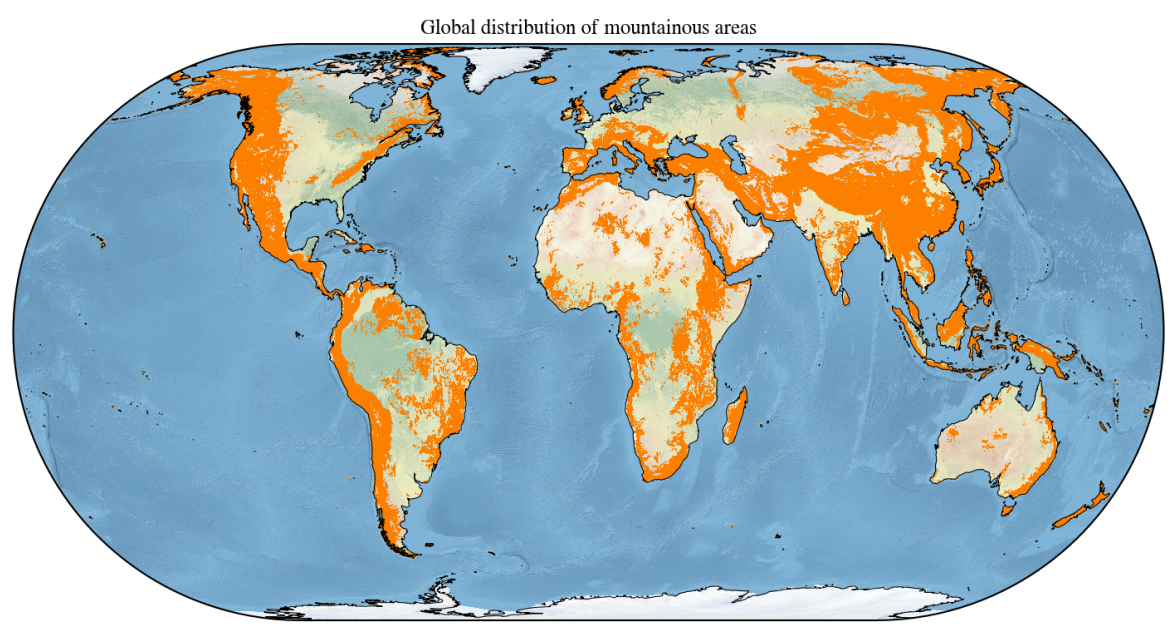

Figure 1: Global distribution of mountainous areas (data source: Global Mountain Explorer).

perspective, the imperfect representation of flow over mountains contributes to systematic model errors in both numerical weather prediction (NWP) and climate simulations. These errors remain one of the major sources of structural uncertainty for Earth-system models despite the ever-increasing resolution.

In recent years, the societal demand for meteorological and climate information has largely increased in knowledge areas beyond meteorology and climatology. End-users and stakeholders require weather forecasts or climate-change projections in sectors such as air pollution science, renewable energy harvesting, hydrology and water resource management, agriculture, ecology, health, and tourism. Meteorological and climatological information referring to mountains is also indispensable in dealing with natural hazards and weather extremes (hail, flooding, droughts) and their impacts, which are relevant not only to the population of mountainous regions (about $10 \%$ of the world's total), but also to that of adjacent low-land areas (a further 15\%).

All these considerations demonstrate the need for increased, internationally coordinated efforts to improve the understanding of exchange processes between the surface and the atmosphere over mountains. TEAMx aims at combining joint experimental and numerical modelling efforts towards better scientific understanding of the mountain boundary layer (MoBL). This may be defined as the part of the troposphere that is directly influenced by the mountainous terrain. More specifically, the MoBL is subject to mountain-induced thermal circulations and venting processes, as well as to mechanical lifting of air masses and the associated buoyancy perturbations in the flow. The MoBL exchanges heat, momentum, tracers and moisture with the Earth's surface and the free atmosphere on time scales between about one hour and one diurnal cycle, and is heavily influenced by meso-scale and synoptic-scale weather processes.

Basic research on the MoBL provides useful knowledge to deal with some of the grand challenges mountain regions worldwide are facing. In a long-term perspective, TEAMx research outcomes will contribute to: (i) reducing uncertainties and systematic errors in 
numerical weather predictions and climate simulations, in particular concerning high-impact weather events over mountains (e.g., flash floods); (ii) understanding the dynamical and chemical processes that determine the exposure of human population and mountain ecosystems to air pollution; (iii) estimating carbon uptake over complex orography more accurately for a better quantitative knowledge of the global carbon cycle; (iv) estimating the vulnerability of mountainous areas to hydrological risk in present and future climate.

Intensive observations of the MoBL have already been carried out in a number of field experiments. Recent campaigns with large-scale deployment of micrometeorological measuring equipment and profiling instruments include T-REX (Owens Valley, California, 2007), MATERHORN (Dugway Proving Ground, Utah, 2012-2013) and Perdigão (2017, Portugal). Data collected during these experiments are by now in the public domain and can be exploited for research targeting the TEAMx goals. However, these experiments privileged either dry mid-latitude environments (T-REX, MATERHORN) or sites with only moderate orographic complexity and scale interaction (Perdigão). Making progress towards understanding exchange processes in orographically complex and relatively moist regions requires carrying out a dedicated field campaign. The European Alps were selected as the optimal target area of TEAMx for a variety of reasons.

First, the Alps are a preferred location to observe exchange processes in an area with extremely complex orography and abundant moisture supply, due to the proximity of the Mediterranean Sea. Compared to other field experiments in the Mediterranean region (e.g., MAP in 1999, or the HyMeX Special Observation Periods in 2012-2013), the plans for TEAMx are to pay greater attention to small-scale atmospheric motions in the planetary boundary layer, and to extend the observation period to a full year in order to comprehensively characterize the seasonal variability of exchange processes.

Second, in the Alps it is possible to carry out intensive observations over extremely diverse surfaces (urban areas, forests, pastures, bare rock, glaciated or snow-covered areas) in relatively short distances. Third, in this region high population density and diverse human and economic weather-sensitive activities make MoBL research relevant to a large variety of stakeholders. Fourth, most of the promoting institutions of TEAMx are based in Europe.

Finally, the dense routine observation network of automatic weather stations, weather radars, FluxNet sites and ceilometers existing in the Alps is unparalleled in other mountainous regions of the world. During the TEAMx experimental phase, these observational facilities will be augmented by ground-based and airborne in-situ and remote-sensing measurement platforms, operated in selected subdomains of the mountain range (e.g., trans-Alpine transects).

The TEAMx modelling activity will include a comprehensive evaluation of the skill of numerical weather prediction and climate simulation codes over complex terrain, but also a sequence of process-oriented model evaluation studies based on idealized simulations with increasing complexity (largely inspired by GABLS, the GEWEX Atmospheric Boundary Layer Study), and a CORDEX Flagship Pilot Study on convection-permitting climate simulation in the Alpine region.

This White Paper provides a concise overview of the outstanding scientific issues and of the expected outcomes of the programme (Tab. 1, Fig. 2). It also outlines the basic elements of a strategy towards the implementation of TEAMx collaborative research, and describes the foreseeable impacts of coordinated experimental efforts. Details on the design of field observations and modelling activities in TEAMx will be provided in the forthcoming Experimental Plan. 


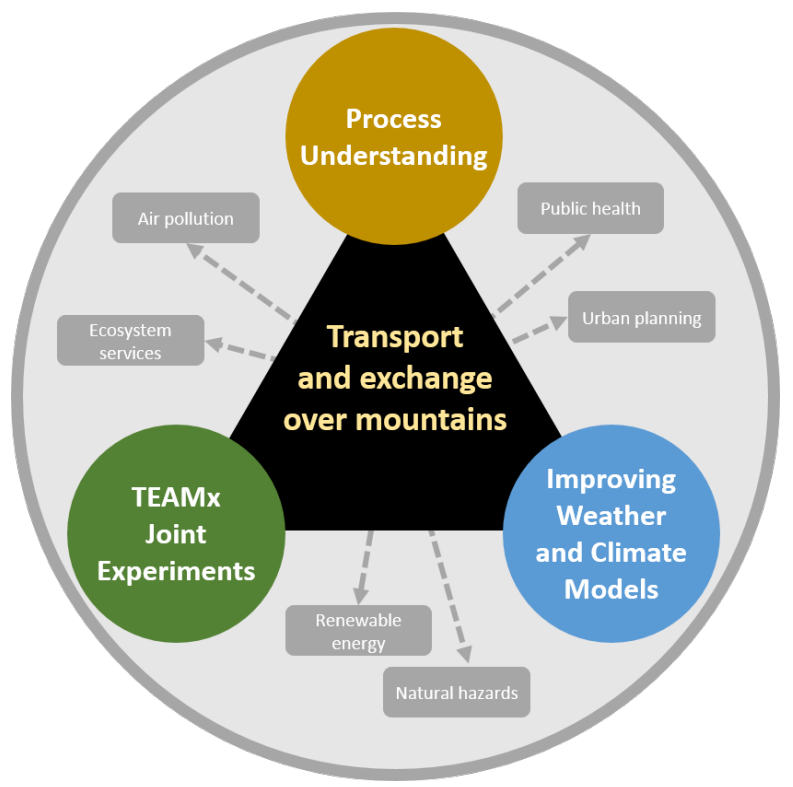

Figure 2: Objectives of the TEAMx programme. Better models, based on better process understanding and on TEAMx data for validation, will lead to more accurate information for weather and climate service providers (grey boxes are examples of application areas).

\begin{tabular}{|c|c|c|}
\hline Objective & \multicolumn{2}{|c|}{ Primary focus } \\
Process Understanding & $\begin{array}{c}\text { Micro- and meso-scale } \\
\text { processes within and above the } \\
\text { mountain boundary layer } \\
\text { (MoBL); Interaction between } \\
\text { scales. }\end{array}$ & $\begin{array}{c}\text { Target } \\
\text { Quantitative understanding of } \\
\text { water, energy and mass } \\
\text { exchange over mountainous } \\
\text { terrain. }\end{array}$ \\
\hline TEAMx Joint Experiment(s) & $\begin{array}{c}\text { Collaborative use of } \\
\text { multi-platform instrumentation } \\
\text { to sample the spatial } \\
\text { heterogeneity of turbulence } \\
\text { and mesoscale circulations } \\
\text { over and near mountains. }\end{array}$ & $\begin{array}{c}\text { Quality-controlled } \\
\text { observational data pool, } \\
\text { available for process } \\
\text { investigation, high-resolution } \\
\text { model verification, } \\
\text { parameterization development. }\end{array}$ \\
\hline Improving Weather and & $\begin{array}{c}\text { Models right for the right } \\
\text { reason, i.e., identification and } \\
\text { reduction of model biases and } \\
\text { uncertainties over complex } \\
\text { terrain. }\end{array}$ & $\begin{array}{c}\text { Weather forecasts and climate } \\
\text { simulations over mountains as } \\
\text { good as over flat terrain, and } \\
\text { less reliant on model output } \\
\text { post-processing. }\end{array}$ \\
\hline Support to Weather and & $\begin{array}{c}\text { Air pollution, hydrology, } \\
\text { climate change scenarios (e.g., } \\
\text { elevation-dependent climate } \\
\text { change) }\end{array}$ & $\begin{array}{c}\text { Smaller uncertainty of impact } \\
\text { models, due to reduced errors } \\
\text { in weather and climate } \\
\text { information. }\end{array}$ \\
\hline Climate Service Providers & & \\
\hline
\end{tabular}

Table 1: Summary of scientific issues and expected outcomes of TEAMx. 


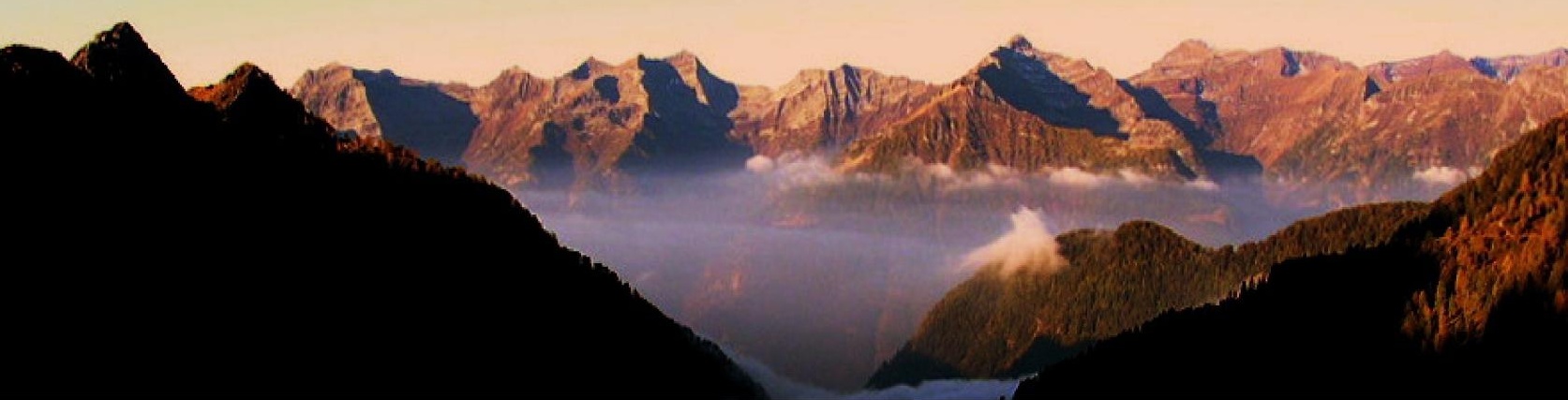

\section{Scientific Objectives}

Exchange processes in the atmosphere include land-atmosphere interactions, as well as transport and mixing within the atmospheric boundary layer and between the atmospheric boundary layer and the free atmosphere. Decades of mountain meteorology research have shown that these processes are more complex over mountains than over flat and homogeneous terrain, in terms of the relevant scales of motion (Fig. 3). It remains unknown (i) whether the larger spectrum of relevant scales of motion generally enhances land-atmosphere exchange over mountains, in terms of spatially aggregated fluxes; and (ii) whether modified exchange efficiency explains the observed stronger sensitivity of mountainous areas to climate change. There is a critical need for novel observations to support theory and model development.

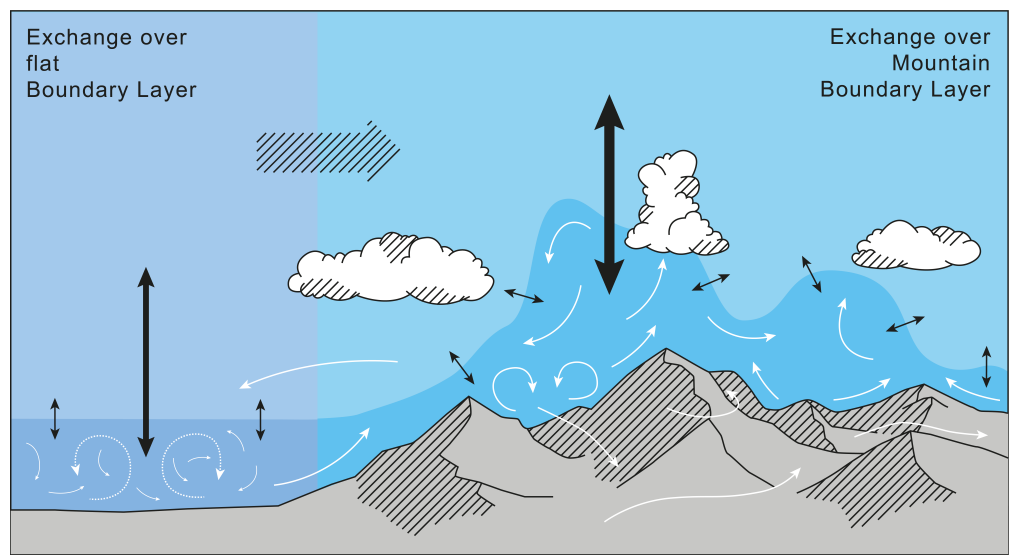

Figure 3: Sketch of exchange processes over mountains. Exchange processes govern the transfer of heat, momentum and mass between the ground, the planetary boundary layer and the free atmosphere. Over complex terrain, they include turbulent mixing, breeze systems, gravity wave propagation and moist convection. Vertical transport towards the free atmosphere is conjectured to be on average more intense over mountains (thicker black arrow). 


\subsection{Understanding exchange processes within and above the MoBL}

The atmospheric boundary layer (ABL) is typically defined as the portion of the atmosphere that responds to variations in surface forcing at time scales on the order of 1 hour or less. A distinctive feature of the ABL is turbulence, which causes effective mixing of atmospheric properties, in particular in the vertical direction. The Mountain Boundary Layer (MoBL) is persistently subject to processes other than turbulence, e.g., thermally-driven local wind systems. These determine complex patterns of variability in three dimensions, which are hard to observe and predict and have a direct impact on surface exchange. Organized air motions also impact exchange between the MoBL and the free atmosphere, in addition to other meso- and synoptic-scale motions (deep moist convection, frontal systems) that cause intermittent deep exchange. This chapter briefly reviews the overall structure of the MoBL and the properties of its lower and upper boundaries (Sec. 2.1.1-2.1.4). It also deals with the phenomena that lead to deep coupling between the MoBL and the free atmosphere (moist convection in Sec. 2.1.5, gravity waves in Sec. 2.1.6). Furthermore, it extends the scope of MoBL research towards climatological time scales (Sec. 2.1.7) and towards connecting dynamical and chemical processes (Sec. 2.1.8-2.1.9).

\section{Relevant literature}

$\rightarrow$ De Wekker, S.F.J. and M. Kossmann (2015): Convective Boundary Layer Heights Over Mountainous Terrain-A Review of Concepts. Front. Earth Sci., 3, 77.

$\rightarrow$ Kirshbaum, D.J., B. Adler, N. Kalthoff, C. Barthlott and S. Serafin (2018): Moist Orographic Convection: Physical Mechanisms and Links to Surface-Exchange Processes. Atmosphere, 9, 80.

$\rightarrow$ Lehner, M. and M.W. Rotach (2018): Current Challenges in Understanding and Predicting Transport and Exchange in the Atmosphere over Mountainous Terrain. Atmosphere, 9, 276.

$\rightarrow$ Pepin, N., R.S. Bradley, H.F. Diaz, M. Baraer, E.B. Caceres, N. Forsythe, H. Fowler, G. Greenwood, M.Z. Hashmi, X.D. Liu, J.R. Miller, L. Ning, A. Ohmura, E. Palazzi, I. Rangwala, W. Schöner, I. Severskiy, M. Shahgedanova, M.B. Wang, S.N. Williamson and D.Q. Yang (2015): Elevation-dependent warming in mountain regions of the world, Nature Climate Change, 5, 424-430.

$\rightarrow$ Rotach, M.W., G. Wohlfahrt, A. Hansel, M. Reif, J. Wagner, and A. Gohm (2014): The World is Not Flat: Implications for the Global Carbon Balance. Bull. Amer. Meteor. Soc., 95, 1021-1028.

$\rightarrow$ Serafin, S., B. Adler, J. Cuxart, S.F.J. De Wekker, A. Gohm, B. Grisogono, N. Kalthoff, D.J. Kirshbaum, M.W. Rotach, J. Schmidli, I. Stiperski, Ž. Večenaj and D. Zardi (2018): Exchange Processes in the Atmospheric Boundary Layer Over Mountainous Terrain. Atmosphere, 9, 102.

$\rightarrow$ Vosper, S.B., Ross, A.N., Renfrew, I.A., Sheridan, P., Elvidge, A.D., Grubišić, V. (2018): Current Challenges in Orographic Flow Dynamics: Turbulent Exchange Due to Low-Level Gravity-Wave Processes. Atmosphere, 9, 361.

\subsubsection{Land-atmosphere exchange}

Measurements of the surface energy balance are often plagued by lack of closure. That is, the sum of the measured sensible, latent and ground heat fluxes is less than the measured net radiation. At ideal sites, lack of closure is often connected to inadequately sampled mesoscale motions, causing significant horizontal and vertical advection. Complex terrain sites are systematically affected by such motions, and therefore often exhibit substantial underclosure. 
Unaccounted advective fluxes are a prominent problem also for $\mathrm{CO}_{2}$ flux measurements, which, integrated to daily and longer term budgets, aim at quantifying the carbon dioxide source/sink strength of the underlying surface. Because advective fluxes appear to make a larger contribution during nighttime conditions, when $\mathrm{CO}_{2}$ is generally emitted from ecosystems, as opposed to daytime conditions, when the active plant canopy absorbs $\mathrm{CO}_{2}$, a potential systematic bias in daily and longer term budgets may arise.

In addition, significant uncertainty in the estimation of land-atmosphere exchange (LAE) derives from the lack of consolidated approaches to handle basic data-processing tasks (as further detailed in Sec. 2.2.1), and from experimental challenges in accurately quantifying all terms of the surface mass balance, in particular inside the canopy layer. A conceptual problem is also the lack of a properly defined reference (null hypothesis) against which measurements can be compared.

Besides the complexity of the terrain height itself, mountainous areas often display dramatic soil and land-cover (vegetation) heterogeneity. Due to changes in elevation, different climate zones occur across short horizontal distances in mountainous environments. As ecosystems adapt to this variability, spatial heterogeneity in ecosystem structure and functioning impacts exchange processes (e.g., through varying soil properties, vegetation, roughness, etc.). Implications include not only marked spatial variability of the measured turbulent fluxes, but also directional variability of the fetch at a single point. It follows that point measurements over complex terrain generally have limited representativeness, making generalizations difficult and compromising their usefulness as ground truth for models.

An important consequence is that, over complex terrain, models of exchange processes are not as well constrained by observations as they are over flat and homogeneous terrain. For instance, no universal scaling behaviour replacing Monin-Obukhov Similarity Theory (MOST) or other scaling regimes has been found over complex orography so far. The limited applicability of existing turbulence theory over complex terrain also stems from frequent violation of its basic assumptions (e.g., stationarity and isotropy of small-scale turbulence).

$\rightarrow$ Increasing efforts should be devoted to understanding the factors that determine the lack of closure of the surface energy balance and the underestimation of the $\mathrm{CO}_{2}$ mass balance.

$\rightarrow$ Progress in theoretical and numerical models of land-atmosphere exchange over complex orography (in particular, flux-profile scaling laws needed in parameterizations) requires generalizing similarity theory by accounting for horizontal heterogeneity in landforms and land-cover.

$\rightarrow$ Surface exchange is heavily affected by the state of soil and vegetation, which can display marked spatial variability. Improved experimental characterization of soil properties and canopy should be pursued, as well as better modelling of their impact on surface fluxes at spatial scales comparable to the grid elements of a NWP model.

$\rightarrow$ In addition to the LAE of vegetated surfaces during summertime conditions, experimental and modelling efforts should be directed towards wintertime conditions and snow-covered surfaces, glaciers, urban areas and water bodies, essential land cover types in mountain areas.

\subsubsection{Three-dimensional structure}

The theory of land-atmosphere exchange over horizontally homogeneous terrain accounts mostly for the role of mixing due to small-scale turbulent processes. A prominent approach in this context is the boundary-layer approximation: turbulent mixing is mostly effective in the 
vertical direction, and horizontal advection can be neglected. Quantitative description of 1D vertical mixing affected by buoyant turbulence production or loss is well rooted in theory (e.g., MOST, see Sec. 2.1.1) and is incorporated in virtually all land-surface-atmosphere parameterizations for NWP and climate models. Other scaling principles based on the boundary-layer approximation (e.g., mixed-layer scaling for the convective ABL, or z-less local scaling for the stable $\mathrm{ABL}$ ) are used extensively in ABL parameterizations.

Where landforms or land cover are spatially heterogeneous, the validity of the boundarylayer approximation breaks down. Baroclinicity due to differential heating or cooling generates local wind systems, which are connected to substantial spatial variability in the boundary layer. The interaction between turbulence and thermodynamically driven mesoscale flows determines both the spatial extent of the MoBL and its efficiency in exchanging energy and atmospheric constituents between the mountainous surface and the free atmosphere. Also the dynamic response to flow over terrain, such as wake formation, creates intrinsic spatial heterogeneity. In these conditions, land-atmosphere exchange is determined not only by vertical mixing, but also by horizontal mixing and three-dimensional advection.

Advective processes have an impact on several aspects of MoBL flow. First, the MoBL responds to surface forcing on time scales considerably longer than one hour (i.e., the commonly accepted scale over flat terrain). Because of the many relevant scales of motion, the quantitative description of the MoBL needs to take into account a multiplicity of time and length scales, some of which depend on geometric properties of the orography (for instance, the scale depth of anabatic and katabatic flow depends on slope angle). The MoBL may contain regions with small levels of turbulence (for instance, daytime mid-valley subsidence areas), or where turbulence is advected instead of being generated locally (for instance, atmospheric rotors). Finally, atmospheric kinetic energy spectra from complex-terrain sites often do not exhibit a clear energy gap between synoptic and turbulent scales of motion-a challenging aspect for ABL parameterizations, which are generally based on evident separation between resolved and unresolved scales.

$\rightarrow$ Experimental evidence of the three-dimensional distribution of turbulence in the MoBL is currently very limited. Novel in-situ and remote-sensing observations and experimental strategies are required to strengthen the existing knowledge, which is mostly based on numerical results (often idealized simulations).

$\rightarrow$ Precise definition of the vertical extent of the MoBL is elusive. Objective methods to determine the spatially inhomogeneous MoBL height must be developed and tested.

$\rightarrow$ Theoretical and modelling work is needed to relate the intensity of thermally driven slope- valley- and plain-to-mountain flows to the respective forcing factors and to quantitative descriptors of orographic variability.

$\rightarrow$ Quantitative knowledge on how thermally-driven orographic flows interact with or are modified by other types of baroclinic mesoscale circulations (e.g., lake and sea breezes) is still largely missing.

$\rightarrow$ In view of improving the existing parameterizations of exchange processes, research should be oriented towards quantifying the upscale impact of turbulence and organized mesoscale motions on the total exchange in the MoBL.

\subsubsection{Exchange in the stable MoBL}

When the atmosphere near the surface is stably stratified due to cooling, exchange between the boundary layer and the free atmosphere is strongly reduced, except for sporadic mixing events caused by high wind shear and internal gravity waves. The latter are particularly important 
because they transport negative momentum from small-scale terrain to the lowermost critical level. The related wave drag can be of the same amplitude of, or even larger than, the turbulent drag.

Mixing in the stable boundary layer (SBL) is observed to be intermittent and affected by sub-mesoscale motions, such as meandering flow, but it is artificially enhanced in surface-layer parameterizations (for instance, using long-tail similarity functions). Intermittent mixing occurs over flat terrain as well, but may be more frequent over complex orography, where both cold air pooling and gravity wave generation are favoured. Over mountains, the likely orographic origin of many sub-mesoscale disturbances affecting the SBL offers some hope of enhanced predictability, at least in a stochastic sense.

SBL processes are particularly important in densely populated mountainous areas. Pollutant emissions in mountains are commonly concentrated at the floor of valleys and basins, where the main urban areas and the major traffic routes are, and where most of the population lives. Under unfavorable conditions, especially in winter and particularly where the surrounding orography impedes cold air drainage, persistent cold air pools may strongly inhibit atmospheric dispersion and expose the population to hazardous pollutant concentrations.

However, urbanized areas have a distinctive impact on surface exchange. The surface energy balance at urban surfaces is affected by reduced nocturnal cooling, which is related to urban heat islands and may induce local circulations that drive air from suburban areas into city centers, favouring ventilation. Reduced cooling also affects the build-up of nocturnal ground-based temperature inversions and their morning breakup, making the urban atmosphere less resistant to mixing. Finally, the high roughness of the urban canopy can generate internal boundary layers. Whether urban canyons are well ventilated or shielded from larger-scale flows largely depends on their orientation with respect to the dominant orographically-induced atmospheric circulations.

Besides urbanization, other types of land-cover variability exert a marked impact on SBL exchange. One key factor in mountainous areas is snow cover. Due to its high albedo, snow reflects most of the incoming solar radiation, helping the maintenance of low near-surface temperatures and potentially affecting photochemical reactions. Albedo evolves with the ageing of the snowpack and is affected by the deposition of particulate matter (black carbon). In most NWP and climate models, the over-simplified treatment of the effect of snow cover on the surface energy balance and roughness is potentially at the root of distinctive wintertime biases. Accurate snowpack modelling is also relevant for climate change simulations, because the snow-albedo feedback is thought to be one of the major drivers of elevation-dependent warming (Sec. 2.1.7).

$\rightarrow$ Coordinated numerical and observational experimentation can help develop better parameterizations of intermittent mixing in the stable MoBL.

$\rightarrow$ Further investigations are needed on the effects of urban meteorological processes on the formation and breakup of nocturnal and persistent cold air pools.

$\rightarrow$ Better modelling of energy and mass exchange over snow-covered surfaces requires not only more complex snowpack models (e.g., multi-layer vs. one-layer), but also improved surface-layer schemes for the SBL.

\subsubsection{Mountain venting}

Over flat terrain and in weak synoptic flow, the upper boundary of the ABL depends primarily on the thermal structure of the atmosphere and on the intensity of turbulence. In dry convective conditions, exchange across the top of the flat-terrain ABL is limited to the turbulent entrain- 
ment caused by overshooting thermals. The related downward transport of potentially warm air from the free atmosphere causes the entrainment layer to be capped by a strong inversion.

In contrast, such a sharp transition is rarely found at the top of the MoBL. Over complex orography, the dry convective MoBL often features a layered structure, in which stable layers (e.g., temperature inversions) intervene between more turbulent elevated mixed layers. Thermal plumes on mountaintops are generally connected to horizontal convergence-divergence patterns responsible for mountain venting. Venting controls the entrainment of free-tropospheric air into the MoBL and, in connection with synoptic advection, provides a powerful mechanism to redistribute heat, water vapour, aerosols and trace gases both vertically and horizontally.

Venting is thought to be extremely sensitive to cloud cover at sunrise. When the condensation level is below mountain top and a low-level inversion impedes vertical mixing, stratus cloud decks over a valley may persist for a long time, inhibiting the onset of upslope winds and largely reducing the turnover of air masses between the valley and the free atmosphere.

Due to venting and horizontal transport and mixing, it is often hard to univocally identify a single mixing height over complex terrain. The upper boundary of the convective MoBL generally does not follow smoothly the shape of the terrain and diagnoses of its height from temperature and aerosol concentration profiles do not coincide.

Due to the absence of sharply defined boundaries between the MoBL and free-tropospheric air, it is challenging to identify the atmospheric layers where mountain venting occurs and to quantify the related mass fluxes. Pathways towards better understanding of mountain venting may include routine measurements of water vapor isotope ratios $\left(\delta \mathrm{O}^{18}\right.$ and $\left.\delta \mathrm{D}\right)$, or other tracers with comparable lifetimes, along vertical profiles (or at a number of vertical heights). These would allow one to characterize air masses that have interacted with the MoBL and to quantify the degree of mixing, even in the case where boundaries between these layers are ill-defined.

The usefulness of isotope ratios stems from the fact that isotopically heavy water molecules are more likely to condense and precipitate, while light molecules are more likely to evaporate. Consequently, the isotopic composition of MoBL air (which typically comprises locally and recently evaporated moisture) and of free tropospheric air (which is depleted of heavy molecules due to progressive rainout on the way to the measurement site) are markedly different. Exchange between these layers, and even the degree of mixing, can be determined by considering the strength of the water vapor isotopic gradient in the vertical.

Moreover, the isotopic composition of water vapor in both the MoBL and free troposphere is itself informative, as it provides strong evidence of the sources of moisture to these layers. This is due, in part, to the facts that different moisture reservoirs are isotopically distinct and that different transport pathways dehydrate air masses in distinct ways. As a result, boundary layer isotope ratios have been used to identify moisture contributions from local evaporation, transpiration, and even urban fossil fuel combustion, while free tropospheric isotope ratios are typically used to distinguish long-distance atmospheric transport pathways and to characterize moisture residence times in the atmosphere. Such information would aid efforts to determine the ultimate source of moisture vented from mountain environments and to evaluate how processes upstream set the background humidity into which MoBL moisture is mixed.

$\rightarrow$ Combined use of in-situ airborne observations and remote-sensing profiling instruments is necessary to observe phenomena at the interface between the MoBL and the free atmosphere in a variety of weather conditions.

$\rightarrow$ Long-term studies of venting and entrainment at individual mountain sites can benefit from continuous measurements of water vapor isotope ratios at two or more nearby 
sites at different altitudes (e.g., along slopes). In principle, such profiles enable one to infer the sources, transport pathways and residence times of atmospheric moisture.

$\rightarrow$ Most of the knowledge on exchange mechanisms at the top of the MoBL is based on numerical studies that neglect synoptic forcing. Interactions with meso- and synoptic scales of motion urgently need to be investigated and covered in the experimental setup.

$\rightarrow$ Coordinated numerical and observational experimentation can help develop better parameterizations of turbulent entrainment and advective transport at the top of the daytime MoBL.

\subsubsection{Boundary-layer control of convective pre-conditioning and initiation}

Flow over and past mountains aids the development of deep moist convection by mechanical (forced) lifting and by generation of thermally-induced circulations. Besides providing the uplift required for convection initiation (CI), heat and moisture exchange in the MoBL and at the surface plays a role in convection preconditioning: it prepares the environment for moist convection by favouring localized humidity build-up, removing convective inhibition and modifying convectively available potential energy. However, exchange processes can also inhibit convection by making the environment less favourable for its onset, particularly through enhanced mixing with dry free-tropospheric air above the higher terrain.

Although the mechanisms of CI and preconditioning related to orographically induced airflow are qualitatively well understood, it remains challenging to quantitatively describe their dependence on the characteristics of the orography. Moreover, the feedbacks of the moist convection on mesoscale dynamic and thermodynamic fields are difficult to understand and predict. This knowledge is required, for instance, to appropriately parameterize the sub-grid-scale orographic forcing for convection initiation in global models, which are not yet convection-permitting and will lack the resolution to adequately capture orographic convection for some time.

On a more fundamental level, correlations and feedbacks between soil moisture and convective precipitation over mountains are still largely unclear, and may be affected both by mountain dimensions and by spatial variability in vegetation and soils (and the resulting soil moisture heterogeneity). Finally, other processes such as katabatic flows, topographicallyinduced internal gravity waves, and other lifting mechanisms may also play a role in the initiation of nocturnal convection near mountains, but the impacts of these processes have yet to be firmly established.

CI is often described as "primary" or "secondary", depending on whether or not preexisting storms provide the necessary lifting for CI. In the former, new storms form in isolation, without the aid of preexisting storms. This mechanism is very common in mountainous regions but remains inadequately understood, in part because it stems from microscale processes that are difficult to observe within the MoBL. In quiescent warm-season flows, primary CI is more common over high terrain during the daytime as up-mountain flows converge over ridges, but it shifts to the surrounding valleys or plains at night as down-mountain flows converge with moist low-level air. Our understanding of Alpine convection development mechanisms would thus greatly benefit of a pan-Alpine climatology of CI (particularly the primary kind), which is currently available only for limited sub-regions.

The Alps are often surrounded by conditionally unstable air masses that are prone to CI if sufficient low-level forcing is provided. Instability in lowlands can occasionally be controlled by venting processes over the adjacent mountains. For instance, advection of elevated mixed 
layers generated by mountain venting can both enhance mid-tropospheric lapse rates and increase convective inhibition, as frequently observed North of the Alps.

South of the Alps, the Po Valley of Italy provides an excellent example of a persistently moist, conditionally unstable and maritime BL, which is occasionally advected by a low-level jet towards the foothills of the Alps. The extent to which this BL flow climbs the Alpine ridge during the day determines whether it participates in primary CI over the high terrain. At night, this layer is perturbed by katabatic flows descending, and transient gravity waves generated by, the Alpine ridge. Thus, the atmospheric conditions of Alpine valleys and forelands, in combination with the local circulation and mesoscale features near the mountains, are important for primary CI during both day and night.

$\rightarrow$ Observations of moist orographic convection should target a broad range of scales, combining high-density in-situ measurements of surface energy exchange (soil moisture, sensible, latent and ground heat fluxes) with retrievals of the wind, temperature, water vapour and precipitation fields from different remote-sensing methods. Observations of atmospheric conditions over both the mountains and the surrounding valleys and plains are important for interpreting CI during both day and night.

$\rightarrow$ Progress in forecasting moist orographic convection crucially depends on reducing initial-condition error at the smallest resolvable scales. This demands upper-air observations of the wind, temperature and moisture fields with higher density and frequency, and improved assimilation of existing and novel high-resolution observations.

$\rightarrow$ To improve the conceptual understanding of orographic convection, hierarchical numerical experiments that span the gap between real-case and idealized simulations, either starting from the latter and moving to more realistic cases or using the opposite approach, should be performed.

\subsubsection{Turbulent exchange due to low-level gravity-wave processes}

Gravity-wave dynamics governs a range of orographic flow phenomena, including föhn, atmospheric rotors and modulation of the stable boundary layer. The impact of these phenomena on near-surface exchange is only partially understood and hard to describe quantitatively.

For instance, a subtle interplay between föhn-induced turbulence and low-level stable layers governs whether föhn reaches low terrain, locally enhancing sensible and latent heat fluxes. The dynamics of föhn breakthrough is recognized to be heavily non-linear, with minor variations in upstream wind and stability profiles potentially causing very different flow responses on the lee side. For this reason, the intensity of downslope windstorms (near the surface) and of the turbulent exchange caused by gravity-wave breaking (aloft) are poorly predictable in general.

Where accelerated föhn flow detaches from the surface, atmospheric rotors can develop; the high levels of near-surface turbulence encountered in rotors are not produced locally, but rather advected from regions of shear instability upstream. Föhn and atmospheric rotors, affected by gaps and peaks in the upstream orography, generally cause sharp horizontal variations (both along and across the flow direction) in the vertical profiles of stability and wind shear, and hence in turbulent transport. Finally, gravity waves can affect the surface energy balance, especially at night, for instance by intermittently dissipating radiative fog. 
$\rightarrow$ Sampling the three-dimensional variability of gravity-wave-driven turbulence requires the coordinated deployment of high-resolution in-situ and remote-sensing measurement platforms, both ground-based and airborne.

$\rightarrow$ Parameterizations of the effects of gravity-wave processes on the large-scale flow typically account for wave momentum fluxes and for turbulent form drag. The available evidence suggests the need to also represent the turbulent fluxes of heat, moisture and other atmospheric constituents that can result either directly from gravity wave breaking, or indirectly from wave-induced enhancement of the wind shear and the resulting dynamic instability.

\subsubsection{Climate change processes and impacts in mountain regions}

Climate change in the mountains is a topic of overwhelming societal and economic value. Its implications are relevant for mountain communities but also for downstream societies, which benefit from the resources and services provided by mountains (primarily, but not exclusively, water). In order to understand how mountains contribute to shaping climate and its evolution, enable more confident projections of future change, and improve mitigation and adaptation strategies for mountain ecosystems and society, progress in four main areas is required:

(i) Improving model parameterizations and reducing model errors. As the global climate warms, mountains are affected by amplified temperature increase, glacier retreat, decreasing extent and duration of snow on the ground and enhanced extreme events at various temporal and spatial scales (e.g., heavy precipitation, heat waves, droughts). The credibility of quantitative projections of these critical changes is limited by factors such as coarse model resolution and imperfect parameterization schemes. These lead to systematic model errors which still need to be better understood and quantified.

Simulating climatic changes requires a realistic representation of climate feedbacks, which partially depend on processes that have to be parameterized in models. Climate feedbacks in mountain environments are mostly related to changes in snow cover and mountain glaciers, clouds and water vapour changes, vegetation dynamics, aerosols. Relevant parameterizations include land-atmosphere exchange models (in particular their snow component), but also microphysics, boundary-layer and convection schemes, which affect the modelling of orographic clouds and precipitation.

Fine-tuning the model representation of mountain exchange processes requires solid observational constraints, which are scarcely available today.

(ii) Improving the mountain environment observational record on climate time scales. Longterm observational capabilities in mountain regions should be improved by increasing the number of in-situ stations that measure essential climate variables, including the surface energy budget components, up to the highest elevations. Integration of insitu measurements with remote sensing observations, particularly form satellite and particularly when in-situ observations are too sparse or available only over very short time scales, would also be beneficial. Global syntheses of observations obtained from current networks and previous studies are necessary to improve physical understanding and to facilitate quantitative evaluation of model performance.

Future long-term observational efforts will have to focus primarily on the temporal and spatial variability of temperature and liquid/solid precipitation (which are key variables due to their influence on the high-altitude cryosphere), but also on all variables affecting the surface energy balance (among others: shortwave and longwave radiation, surface albedo, specific humidity, clouds, aerosols and soil moisture). 
(iii) Advancing the knowledge of the processes which determine mountain climate. One main question is whether mountain environments are warming faster than the lowlands or, more generally, whether warming rates are different at different elevation bands (so-called elevation-dependent warming, EDW). According to the current understanding, EDW is largely determined by feedbacks in which surface exchange processes play a central role (e.g. snow-albedo feedback, cloud-radiation feedbacks). For instance, changing surface albedo (particularly in connection with snow cover changes) turns out to be a main driver of positive EDW (enhanced warming rates with elevation) in several mid- latitude mountain areas, such as the Alps and the Himalayas.

Evidence for elevation-dependent climate change signals in other variables, such as rainfall, snowfall or wind, is less studied than for temperature, partly because of the lack of measurements. In general, evidence for elevation-dependent climate change is not equally conclusive in all mountain ranges worldwide and the extent to which regional differences in elevation-dependent trends depend on the global circulation is little explored.

Recent studies suggest that changes in the large-scale atmospheric circulation have an important impact on climate extremes over mountain ecosystems at least in Europe. Making further progress requires analysing the connection between altitudinal trends of temperature, precipitation or snow cover and large-scale dynamics. A topic of interest is how extreme weather occurrences over the mountains (e.g., the European Alps) are affected by the surface temperature of nearby seas (e.g., the Mediterranean) or by the phases of teleconnections (e.g., the North-Atlantic Oscillation).

A final remark is that the evolution of mountain climate contributes to global changes in similar ways as large ice caps and high-latitude areas do, for instance through loss of ice mass from glaciers and through permafrost thaw. The former process affects sea-level rise and the availability of local water resources, being one of the key Grand Challenges in the WCRP and one of the key topics of IPCC's recent Special Report on the Ocean and Cryosphere in a Changing Climate. The latter is of utmost importance due to its connection to the global carbon cycle and to its potential for significant positive feedback in the climate system, in addition to its direct impacts on human activities.

(iv) Improving capabilities to predict impacts to the mountain environment. The consequences of high-impact weather on mountainous areas are multi-faceted. They include flooding and landslides, which cause loss of life and infrastructure; windstorms, knocking down trees over large areas; more abundant freezing rain on infrastructures such as roads, electric cables and renewable energy plants (cold weather at mid-latitudes is not going away with warming, but weather systems will be far more water-loaded).

Performing impact studies and addressing user and societal needs generally requires enhancing the spatial and temporal resolution of global and regional climate model output by means of statistical downscaling methods. In their various forms, these have the capability of adding considerable value to raw climate projections. Besides statistical approaches, downscaling research should invest in the advancement of physicallybased methods. This may involve the further development of intermediate complexity models, which are not based on numerical integration of the time-dependent governing equations but on simple steady-state models of airflow over mountains and orographic precipitation.

The processes affecting mountain climate directly and through feedbacks include surface-atmosphere interactions, differential warming rates with elevation, extreme 
events, loss of ice mass and physical changes in mountain ecosystems. In addition, the climate of specific mountainous areas responds in largely unknown manners to changes in the global circulation.

$\rightarrow$ Long-term observational capabilities in mountain regions must be enhanced by evaluating new observational techniques for mountain climate networks, remote sensing products for mountain climate applications, and new strategies for network design and data assimilation in complex terrain.

$\rightarrow$ Lack of appropriate observations of past and present climate and poorly parameterized processes in climate projections cause significant uncertainty of climatic trends in mountain regions. Reducing the uncertainty of mountain climate change requires implementing better parameterizations of mountain-induced exchange into models that work on climatological time scales, especially regional/global climate and earth system models.

$\rightarrow$ Knowledge of systematic model errors arising from parameterized processes (e.g., the components of the surface energy balance) in mountainous areas, as well as their dependence on model resolution, is still largely missing and urgently needs to be established.

\subsubsection{Aerosol, trace gases and air pollution}

Atmospheric chemistry in mountainous regions is made complex by the interplay between dynamical effects and a diverse precursor mix of ozone and aerosol-forming compounds.

Studies of ozone chemistry show that air chemistry models tend to underestimate ozone concentrations across the Alps, most likely due to uncertain precursor emissions and associated biases in the VOC (volatile organic compounds)-to- $\mathrm{NO}_{x}$ loading. A question that arises is whether Alpine valleys can nowadays generally be characterized as a mid- to high $\mathrm{NO}_{x}$ and high BVOC (biogenic VOC) environment, and whether ozone production is $\mathrm{NO}_{x}$ or VOC sensitive.

The fate of near-surface emissions, both natural and anthropogenic, also crucially depends on near-source chemical transformations, which are in turn affected by transport and exchange processes. Multi-methodological observational studies usually find significant spatial variation of air pollutants in Alpine valleys during winter, in both the western and eastern Alps. Recently, it was found that high emissions of anthropogenic nitrogen oxides in the Alps seem underrepresented in emission inventories. These spotted observational studies point towards the need of a comprehensive characterisation of gas-phase precursors that fuel ozone chemistry and can form secondary organic aerosols (SOA).

From an air quality perspective, a connected emerging topic is the production of ultrafine secondary aerosols in mountainous regions, which is poorly understood. It has recently been suggested that under certain conditions mountains can act as a source of SOA. While it is well established that particle nucleation events are triggered by the oxidation of anthropogenic or biogenic precursor gases, it is not known at this point whether these events preferentially happen under weak synoptic forcing and to what extent recirculation mechanisms in mountains are playing a role. Since ultrafine particles are observed along mountain slopes and on mountain ridges, orographic processes can have a significant influence on the mesoscale re-distribution of aerosols and their precursor gases.

In mountainous terrain, inhibited vertical exchange (e.g., trapping of pollutants in valleys) leads to local pollution episodes, while enhanced vertical exchange between the surface and the free atmosphere contributes to regional pollution episodes (e.g., build-up and long-range transport of ozone due to fuelling of precursors from the ground). Heavy aerosol pollution 
events can also be sporadically observed due to horizontal advection of air masses into narrow valleys. It is conceivable that aerosols could contribute to elevation dependent warming and alter photolysis rates under these types of conditions.

$\rightarrow$ New observations of the chemical composition in mountains should be conducted to improve the understanding of the interaction between dynamics and chemistry on relevant scales.

$\rightarrow$ A better understanding of sources and chemical processing of aerosols and their precursors should be developed through the planning of targeted field campaigns.

$\rightarrow$ Chemical remote sensing and regional atmospheric models approach the spatial scale to resolve important processes in complex topography - these will help to develop appropriate parameterizations for large scale models.

$\rightarrow$ Long-term chemical atmospheric observations in the Alps should address significant gaps in the evolution of air pollution sources.

\subsubsection{Stratosphere-troposphere exchange}

The ozone in the atmosphere is a critical parameter for climate, air quality and the for stratospheric ozone layer. Its concentration is determined by the chemistry of the atmosphere, by emissions from the surface and by transport. Notable in this is the two-way transport of compounds between the troposphere and the stratosphere. Ozone transported from the troposphere into the stratosphere is a critical term in determining the concentration of ozone in the troposphere and its impact on human health and climate; the transport of CFCs and their replacement into the stratosphere is critical for determining stratospheric ozone loss; the transport of halogen compounds (from the degradation of CFC and their replacements) from the stratosphere is important for determining the concentration of halogens in the upper troposphere.

This transport between the troposphere and the stratosphere is thought mainly to occur due to flow within mid-latitude frontal system and from large convective systems. However, there are some observational studies that have highlighted the potential for breaking gravity waves, induced by flow over mountainous terrain, to also lead to rapid mixing between air masses. This potentially provides an additional, and essentially unquantified, route to mixing air between the troposphere and stratosphere. Most models (unless run at a very high spatial resolution) do not explicitly resolve convective overturning due to gravity wave breaking. For critical meteorological parameters, such as momentum, parameterizations exist to represent the impact of gravity wave breaking on vertical transport. However, they have not (nor are they likely suitable) be applied to the transport of chemical tracers. Thus the role of mountain induced gravity wave breaking on the chemical composition of the atmosphere is at this point unknown.

$\rightarrow$ New observations of the chemical composition within breaking mountain induced gravity waves should be obtained to allow case studies to be developed.

$\rightarrow$ Turbulent transport of chemical tracers induced by breaking gravity waves should be parameterized in chemical transport models.

$\rightarrow$ Models using these parameterizations should be compared to the new observational dataset to evaluate their veracity, then used to explore the role of breaking gravity waves on the composition of the atmosphere, notably the flux of compounds between the stratosphere and the troposphere. 


\subsection{Measurement of MoBL processes}

Measuring microscale and mesoscale transport and exchange processes in the atmosphere over mountainous terrain poses great challenges, due to the large degree of spatial heterogeneity that needs to be sampled with sufficiently high resolution over sufficiently large areas. This requires coordinated use of in-situ and remote-sensing measurement platforms.

Generally, turbulent fluxes are not measured directly, but rather estimated from highfrequency measurements of field quantities. This is true both for turbulence-resolving in-situ measurements (Sec. 2.2.1) and for remote-sensing retrievals (Sec. 2.2.2). In either case, flux estimation relies on assumptions and methodologies whose applicability over complex orography is questionable. The limitations of fixed measurement platforms can be partially overcome by airborne systems (Sec. 2.2.3).

The material in the following sections focuses exclusively on conceptual problems related to the processing and interpretation of measurement data. Practical considerations on the concrete planning of field activities (where, what and how to measure) are given in the upcoming TEAMx Experimental Plan.

\section{Relevant literature}

$\rightarrow$ Emeis, S., Kalthoff, N., Adler, B., Pardyjak, E., Paci, A., Junkermann, W. (2018): High-Resolution Observations of Transport and Exchange Processes in Mountainous Terrain. Atmosphere, 9, 457.

\subsubsection{Turbulence data processing}

The standard approach to evaluate turbulent fluxes is to use a sonic anemometer to measure the three wind components and (sonic) temperature, plus sensors allowing high-frequency measurements for other scalars, followed by application of the eddy covariance method.

For complex terrain applications, possible anemometer tilt and local streamline deformation need to be accounted for by rotating the coordinate system that defines the wind components. The choice of the coordinate system has a non-negligible impact on flux estimation. Additional complications arise if the aerodynamic characteristics of the upwind terrain vary with upwind direction; sector-dependent processing algorithms have been developed to handle these cases.

Also the separation of turbulent fluctuations from the mean flow properties is handled with a variety of methods. This task is complicated by the fact that atmospheric processes over complex terrain seldom attain steady states and, as a consequence, the appropriate cut-off timescale cannot be easily determined. Despite extensive testing, there is no general consensus on a single best approach for all sites and conditions yet.

In the case of stable boundary layers, propagating internal gravity waves can contaminate eddy covariance measurements, so the ability to disentangle turbulent fluctuations from other kinds of atmospheric motions is essential for an accurate estimation of fluxes. Several methods to distinguish wave motions from turbulence were suggested, considering their differences with respect to dominant wavelengths or frequencies, energy transport speed, mixing efficiency, ability to transport scalar quantities, correlations between pressure, temperature, and wind speed fluctuations, and potential vorticity. In practice this is an unsolved issue, and no straightforward and universally accepted approach to the problem exists at present.

Alternative approaches to obtain turbulence information include for instance the use of wavelet transforms in data processing. This method has been used to study canopy flows and airborne turbulence measurements, but its potential for micrometeorological research over 
complex terrain is largely unexplored. Nowadays, measurement principles alternative to the eddy covariance method are available too (e.g., scintillometry, see Sec. 2.2.2).

$\rightarrow$ Progress in understanding turbulent exchange over complex terrain with the eddycovariance method depends on thorough understanding of the implications of data processing (e.g., anemometer tilt correction and the determination of the turbulence averaging scale) on the estimate of turbulence statistics.

$\rightarrow$ Collaborative research systematically testing and comparing alternative approaches to measure or process turbulence data is mandatory.

\subsubsection{Surface-based remote sensing}

The spatial heterogeneity of the atmosphere over complex terrain limits the representativeness of in-situ measurements to relatively small spatial and temporal scales. Surface-based remote sensing can enhance measurements in terms of both temporal resolution (e.g., vertical temperature and humidity profiles from passive microwave radiometers at intervals of minutes) and spatial coverage (e.g., three-dimensional wind fields from scanning Doppler lidars). Nowadays, the increased commercial availability of these technologies provide potential for routine measurements. Another available option to extend measurement coverage is scintillometry, which yields spatially averaged turbulent fluxes in the surface layer and is hence potentially interesting for model verification.

Despite this progress, current remote sensing technologies have only limited ability to resolve small-scale spatial heterogeneities. For example, the vertical resolution of microwave radiometers is insufficient to observe complex vertical structures in valley atmospheres, such as shallow elevated inversion layers.

Besides resolution, even other factors may restrict the applicability of remote sensing techniques over complex terrain. In some cases, the basic assumptions on which the measurement principles rely are not necessarily valid in this setting. One example is that horizontal homogeneity has to be assumed for the derivation of three-dimensional wind vectors from conical Doppler lidar scans, as well as for the boundary-layer scans done with passive microwave radiometers to improve the vertical resolution of temperature profiles in the lower atmosphere. Another example is that turbulence characteristics derived from scintillometer measurements explicitly assume the validity of MOST.

Despite their limitations, remote sensing observations provide new means for determining turbulence characteristics on a larger scale than the point measurements from traditional eddy-covariance systems. However, only few studies have determined profiles of turbulent fluxes so far, which require simultaneous measurements with a combination of instruments, e.g Doppler lidars and a Raman lidar or Differential Absorption Lidar (DIAL). Making progress depends on successful solution of a few technical challenges, partly similar to those affecting turbulence-resolving in-situ measurements (e.g., definition of an appropriate coordinate system and averaging time; see Sec. 2.2.1).

$\rightarrow$ A thorough evaluation of the use of surface-based remote sensing in complex terrain is necessary, including a quantification of potential errors produced by invalid assumptions, such as horizontal homogeneity.

$\rightarrow$ Capturing small-scale mountain-specific processes (e.g., slope winds) and determining turbulence characteristics with surface-based remote-sensing instruments requires a coordinated and optimized strategy based on multiple instruments. 


\subsubsection{Airborne measurements}

Adequate sampling of the spatial and temporal variability of exchange processes over mountains requires the ability to cover large areas. Remote-sensing observations from satellites would in principle be an option, but geostationary satellite observations do not have sufficient horizontal and vertical resolution while orbiting satellites do not allow continuous monitoring. An effective way to sample the three-dimensional spatial variability of the MoBL with adequate resolution and coverage is to perform in-situ measurements with a variety of airborne platforms.

Airborne platforms include balloons (radiosondes), tethered balloons, remotely-piloted aircraft systems (RPAS; also known as unmanned aerial vehicles, UAV) and aircraft.

Most of them are only partially suited for operation in the MoBL. For instance, the typical frequency of release of radiosondes, even during intensive observation periods, usually allows only very coarse temporal resolution. Large aircraft have comparatively limited manoeuvrability and are not designed for low-elevation flight, while also being subject to restrictions on operation imposed by air traffic control for safety reasons (e.g., flight allowed only above a certain altitude). Their operation is costly and thus limited to short field campaigns. Usability of RPAS is excellent for the purposes of vertical profiling and sampling horizontal transects over short distances, but is otherwise limited by their endurance, their ability to carry only small payloads and the requirement of special flight permits. Small aircraft, ultra-light or motor gliders might be a more appropriate solution, provided they can carry enough payload.

$\rightarrow$ Stacked horizontal flights of small aircraft or RPAS are a possibility to obtain spatially distributed measurements of mean quantities as well as turbulent flux profiles. Combined use of several airborne platforms is necessary and requires detailed evaluation of operation strategies, possibly with dedicated campaigns.

$\rightarrow$ Limitations in the processing and interpretation of airborne turbulence measurements, due to inhomogeneity along flight legs, should be taken into account. Data processing should also correct any interference introduced by the measuring platform. 


\subsection{Numerical modelling of the atmosphere over complex terrain}

The ability of atmospheric models to represent mesoscale phenomena and the impact of finescale terrain depends critically on model resolution. Current operational weather prediction limited-area models (LAMs) are non-hydrostatic and adopt a grid spacing on the order of 1 $\mathrm{km}$. Most regional climate models, on the other hand, still adopt hydrostatic dynamical cores and a grid spacing on the order of $10 \mathrm{~km}$. Model resolution determines not only the magnitude of numerical errors, but also the applicability of parameterization schemes, which are typically designed with a specific range of grid spacing in mind.

Factors limiting the skill of high-resolution forecasts include the lack of mesoscale detail in their initial conditions, the fast growth of small-scale forecast errors, and the rapid downscale propagation of large-scale initial errors. Consequently, an important pathway for the advancement of high-resolution weather forecasts is the improved specification of boundary and initial conditions, a task that entails special challenges over complex terrain (Sec. 2.3.1 and Sec. 2.3.2).

Regardless of their resolution, atmospheric models rely on parameterization schemes, which generally oversimplify the atmospheric behaviour over orography, or are empirically tuned to flat-terrain observations. Deficiencies in parameterizations (Sec. 2.3.3) are the cause of systematic model errors, limiting the quality of both short-range weather forecasts and climate simulations regardless of model resolution. In the latter case, the impact of even small model biases is potentially amplified by the very long model integrations. Flawed parameterizations, in combination with imperfect initial conditions, are also a source of uncertainty for LAMs (Sec. 2.3.4).

Atmospheric models are an essential tool not only in weather forecasting, but also in atmospheric dispersion studies over mountains. Modelling transport and diffusion over complex terrain requires both high model resolution and improved turbulence parameterization schemes (Sec. 2.3.5).

\section{Relevant literature}

$\rightarrow$ Chow, F.K., C. Schär, N. Ban, K.A. Lundquist, L. Schlemmer and X. Shi (2019): Crossing Multiple Gray Zones in the Transition from Mesoscale to Microscale Simulation over Complex Terrain. Atmosphere, 10, 274.

$\rightarrow$ Giovannini, L., D. Zardi, E. Ferrero, T. Karl, M. Rotach, C. Staquet, S. Trini Castelli (2019): Atmospheric pollutant transport over complex terrain: challenges and needs for improving air quality measurements and forecasting. Atmosphere (in preparation).

$\rightarrow$ Hacker, J., C. Draper, and L. Madaus (2018): Challenges and Opportunities for Data Assimilation in Mountainous Environments. Atmosphere, 9, 127.

$\rightarrow$ Sandu, I., A. van Niekerk, T.G. Shepherd, S.B. Vosper, A. Zadra, J. Bacmeister, A. Beljaars, A.R. Brown, A. Dörnbrack, N. McFarlane, F. Pithan and G. Svensson (2019): Impacts of orography on large-scale atmospheric circulation. npj Climate and Atmospheric Science, 2, 10.

\subsubsection{Representation of the surface}

NWP models have historically been developed using curvilinear grid systems in which the lower boundary coincides with the Earth's surface. Such terrain-following grids allow for a simple specification of the surface boundary conditions, while making the formulation of the governing equations only moderately more complex. At km-scale resolutions, traditional terrain-following grids encounter problems in resolving correctly the pressure gradients near steep orography, making models more prone to error and numerical instability. Non- 
conforming grid systems, in which the model grid is not transformed to adjust to the terrain, are an area of ongoing research: immersed-boundary and embedded-boundary (or cut-cell) methods, whose use is commonplace in engineering, have been recently introduced into NWP codes. However, their development has only focussed on micro-scale simulations and is still at an experimental stage due to difficulties in specifying surface boundary conditions in high-Reynolds-number flow.

Challenges determined by the increasing resolution of LAM are not limited to the specification of model orography and to the related implications on the grid design. Accurate specification of high-resolution surface boundary conditions depends on the availability of sufficiently resolved global databases of surface properties. While this precondition is currently met for digital elevation models, it is not for other type of land data (e.g., roughness length, land-use, vegetation cover). Even when high-resolution land information is available (e.g., the CORINE database in Europe), it is not straightforward to map categorical land information into physical soil properties (required in land-atmosphere exchange models), and to aggregate high-resolution information to the scale of the grid. The operational monitoring of the soil moisture and temperature fields is also suboptimal: especially in complex terrain, the monitoring relies on sparse and poorly representative observations and on low-resolution remote-sensing retrievals (see also Sec. 2.3.2).

In addition, there is some uncertainty about the most appropriate strategy for specifying surface fluxes over sloping terrain, both in terrain-following grids and in Cartesian grids with immersed or embedded boundaries. Most operational models (based on terrain-following grids) only specify the vertical flux components, in accordance with the boundary-layer approximation. Increasing evidence of the relevance of horizontal fluxes call for a revision of the current paradigm, at least over complex orography. Theory developed from observations over flat terrain (MOST) may not be applicable over complex topography either (see also Sec. 2.1.1).

$\rightarrow$ Immersed-boundary and cut-cell methods show promise to improve the forecast accuracy of near-surface atmospheric fields, but require further research directed to improving the specification of surface momentum and energy fluxes.

$\rightarrow$ Due to the extreme spatial heterogeneity of the physical properties of the land surface in complex terrain, accurate specification of surface boundary conditions is not possible in the absence of high-resolution information and of effective flux aggregation methods.

$\rightarrow$ Near-surface turbulence is often of non-local origin over complex terrain. Hence, the development of non-local parameterizations of surface fluxes is necessary. Such schemes should incorporate information about the lateral variability of physical land properties, like surface roughness or soil moisture, over spatial scales determined by the orography.

\subsubsection{Initial conditions}

The specification of the initial state of NWP model integrations relies on data assimilation (DA). Some of the basic assumptions of modern DA approaches (e.g., small departures between observations and background field; Gaussian error statistics; unbiased model and observations) are hardly met over mountains.

Systematically large departures from the background (due to model biases, unresolved processes or poor observation representativeness) can cause observations in complex orography to be frequently discarded from the assimilation process. In addition, the models of the observation error covariance needed in variational DA methods can prove inaccurate over 
mountains. Potential consequences include fitting observations more closely than would be appropriate; or rejecting observations because of large departures (too large relative to an underestimated background error). Ample margin for progress in both variational and ensemble DA still exists, in particular regarding the assimilation of observations critical for surface exchange modelling (e.g., turbulence and soil moisture).

$\rightarrow$ Examining analysis increments from DA over mountains allows highlighting systematic biases in observations and/or models, offering useful information to improve ABL and land-atmosphere exchange parameterizations over mountains, and consequently DA products themselves.

$\rightarrow$ Improved mesoscale analyses of the atmospheric state may result from coupled DA. In mountainous areas, high-resolution land-only DA, for instance with distributed hydrological models, may be a useful alternative to low-resolution coupled DA. This conjecture is based on the high degree of correlation between atmospheric and land variables often found over mountains (e.g., between atmospheric temperature and soil temperature, or between atmospheric humidity and soil moisture).

\subsubsection{Parameterizations of sub-grid-scale processes}

Atmospheric processes that occur on spatial scales too small to be captured by a model's computational grid are parameterized. Parameterized processes in NWP and climate simulations include boundary-layer turbulence, land-atmosphere exchange, radiative transfer and, depending on the model resolution, orographic drag, shallow and deep moist convection. A parameterization represents the bulk effects of a sub-grid-scale process using simplified models that rely on the resolved quantities. As the horizontal resolution of atmospheric models steadily increases with time, the explicit representation of atmospheric processes of progressively smaller scale becomes feasible. Following this trend, traditionally parameterized processes (e.g. moist convection, turbulence) become at least partially explicitly resolved. However, for any given process, there is no sharp resolution limit between explicit representation and mandatory parameterization. The range of model resolutions that lies between the large-scale limit beyond which parameterization is warranted and the small-scale limit beyond which the process is fully resolved on the model grid is referred to as the grey zone.

Parameterization schemes are usually developed with a specific grid resolution in mind, that is, they are not designed for a proper transition across the grey zone. For instance, the underlying logic of ABL parameterizations for RANS mesoscale NWP models largely differs from that of turbulence parameterizations for LES models. In the current modelling paradigm, high-resolution simulations are only made in nested domains, where specific sub-grid-scale models designed for small grid spacing are adopted. In fact, this is a pragmatic way of avoiding running models at grey-zone resolutions. More often than not, fine-scale structures in nested domains do not develop in a realistic manner, because they cannot be properly initialized and advected from the outer domains, and they are affected by spurious wave motions originating along the lateral boundaries. The limitations of current parameterization schemes, especially of turbulence and convection, call for further investigations on improving their scale adaptivity.

Besides grey-zone issues, boundary-layer and surface-layer parameterizations fundamentally suffer from oversimplified treatment of horizontal exchange (for instance: purely local and parameterization of vertical land-atmosphere exchange or, in the case 1.5-order ABL schemes, neglecting horizontal derivative terms in the turbulent kinetic energy equation).

Inaccurate parameterizations of SGS processes can introduce distinctive model biases. These can be aggravated by extended integration times, e.g., in climate modelling. For instance, 
latent heat exchange modelling based on concepts for flat terrain may not decisively deteriorate a weather forecast, but it might systematically yield too much or too little evaporation, so that a particular location (not necessarily only the one affected by the largest evapotranspiration bias) gets too dry or too wet over the years of a climate simulation, altering the meso-scale flow patterns and feeding back to the larger-scale flow.

Another example relates to orographic drag. Climate models suffer from biases in the hemispheric circulation due to an imperfect treatment of orographic drag (see also Sec. 2.1.6). The partitioning between parameterized and resolved orographic drag varies greatly between different models, as it is mostly determined by ad-hoc tuning of the parameterization schemes. The same concept applies to the partitioning between gravity-wave drag and form drag.

These shortcomings are known because, due to their obvious impact on the global circulation, modelled orographically-induced momentum fluxes have undergone careful scrutiny. Knowledge on the global-scale effect of orographically-induced energy and mass fluxes, and on the adequacy of their parameterization in models, is instead largely missing.

$\rightarrow$ While the biases related to the modelling of momentum exchange over mountains have been studied extensively, justifying the urgency of improved orographic drag schemes, the possible impact of imprecise modelling of heat and moisture exchange over mountains has so far eluded investigation.

$\rightarrow$ Future research should be devoted to testing and improving scale-adaptive parameterizations, especially unified mixing schemes that represent simultaneously the local effect of turbulent mixing and the non-local effect of convective and terraininduced transport. The further development of nesting methods that allow the realistic development of fine-scale structures in high-resolution grids should also be pursued.

$\rightarrow$ The bulk relationships contained in parameterization schemes are commonly valid under very restrictive assumptions on the properties of SGS motions (e.g., stationarity, horizontal homogeneity). For this reason, semi-empirical closures generally explain only a small fraction of the variance of experimental data. From a data analysis perspective, the correlation between predictors (resolved quantities) and predictands (parameterized quantities) can be considerably improved using machine learning (or artificial intelligence) algorithms. Machine-learning-based parameterization schemes are still in their infancy, and great potential for their further development lies in complex-terrain micrometeorological observations, which often deviate from simple theory.

$\rightarrow$ For similar reasons, machine learning algorithms can be profitably used also in the post-processing and downscaling of NWP and climate simulations, especially in complex-terrain scenarios.

\subsubsection{Forecast uncertainty and atmospheric predictability}

Errors in initial conditions, numerical approximation, imperfect parameterization schemes and non-linear error growth are the primary sources of uncertainty in numerical weather predictions. Forecast uncertainty evolves during the forecast range and, in practice, it is generally estimated with ensemble prediction systems (EPSs). The first operational ensemble forecasts were based on global models and date back to the early 1990s, but limited-area ensembles with convection-permitting grid spacing gained increasing popularity in more recent years.

Future increments in available high performance computing resources can be invested in increasing the number of ensemble members, or their resolution, or both. Larger ensembles 
would in principle allow a better sampling of forecast probability density functions, while higher-resolution ensembles would permit the explicit modelling of a larger range of mesoscale weather phenomena, including the orographically forced ones (e.g., orographic convection and mountain wave drag). Studies on which of the two alternative approaches should be prioritized are still scarce.

Limited-area ensemble forecasting has its own specific challenges. First, handling of lateral boundary conditions is an additional source of uncertainty in this case. In addition, it remains unclear whether the various perturbation methods used in convection-permitting ensembles are equally effective as those adopted for global EPS. Recent scale-dependent predictability studies suggested that outputs from individual members of convection-permitting ensembles become fully de-correlated at the smallest resolved spatial scales in a matter of only a few hours. This implies low practical predictability at the small scales, potentially due to a perturbation design that is not sufficient to fully model the initial-condition uncertainty.

Uncertainty due to imperfect model formulation is estimated in EPSs using model error schemes. These schemes, which primarily serve the need of inflating ensemble spread, are presently based on the introduction of spatially and temporally correlated stochastic perturbations either into the parameterized tendencies of prognostic variables or in the least well-constrained free parameters of the parametrization schemes. However, the current understanding of the dynamical impact of these perturbations remains rather limited.

NWP ensembles are also useful in the evaluation of deterministic parameterizations, because they permit quantifying the contribution of model error to the total forecast uncertainty and, therefore, the extent to which the latter can be reduced by developing better models of sub-grid-scale effects.

$\rightarrow$ Predictability issues in convection-permitting EPS are not specific to complex-terrain modelling, yet relevant to the context. On the one hand, it can be intuitively expected that explicitly resolved large-scale orographic forcing is a source of predictability. On the other hand, orographic forcing is also effective on the meso- and micro- scales of atmospheric motion, which are typically poorly initialized and may cause rapid error growth and large uncertainty. The weather conditions and the spatial and temporal scales at which orography effectively enhances atmospheric predictability are not known in detail.

$\rightarrow$ Useful developments of stochastic parameterizations of turbulent mixing and moist convection may result from physically based and regime-dependent schemes aware of sub-grid-scale orographically-induced air motions (e.g., inherently stochastic treatment of surface-layer exchange over non-homogeneous terrain, as opposed to universal use of MOST).

\subsubsection{Pollutant transport modelling}

At present, the optimal architecture of dispersion models for applications over complex terrain consists of coupled high-resolution meteorological simulation and Lagrangian Particle Dispersion Models (LPDM). LPDM are preferred to Eulerian models because, if properly generalized to account for horizontal exchange, in principle they allow better simulation of plume rise in the near-source region and where the assumption of horizontal homogeneity cannot be made. Sub-km grid spacing in the meteorological simulations is necessary in order to make the effective model resolution enough to properly represent small-scale orography and its impact on the mean flow. 
While technically feasible, sub-km resolutions lie within the grey zone of ABL parameterization schemes. This implies that any property of the sub-grid-scale turbulence used to inform LPDMs may be only poorly representative. Skipping the grey zone is possible in research applications by coupling LPDM and LES, but is unfeasible for operational purposes. For the latter, progress in turbulence parameterization design and in the determination of scaling parameters (such as the MoBL height) seems to be mandatory. A further challenge lies in accounting for chemical reactions, especially those with high-order kinetics, in LPDMs.

$\rightarrow$ High-resolution atmospheric models coupled with LPDM are presently the most appropriate tool to simulate pollutant dispersion. Application over complex terrain requires good-quality mean flow and turbulence fields from the meteorological component, as well as LPDM that account for horizontal exchange.

$\rightarrow$ Further research on practical ways to represent the fate of reactive tracers in LPDM is necessary.

$\rightarrow$ Tracer experiments conducted at sites with different degrees of orographic complexity are needed to support model validation efforts, in particular ensemble approaches. 


\subsection{Expected impacts}

\subsubsection{Better understanding}

The practical aim of weather forecasting may be considered to meet sufficient quality standards in the forecasts of basic weather elements (precipitation, temperature, wind). However, many other atmospheric variables related to turbulence and exchange processes are routinely considered in applied meteorology (Sec. 2.4.3). Examples include surface heat exchange, turbulence kinetic energy, Reynolds stresses and turbulent fluxes throughout the ABL. Typically, these quantities are not explicitly resolved by NWP models, but parameterized. Simulations of these quantities are not routinely verified, but rather tuned in order to optimally reproduce empirical evidence from temporally and spatially limited observational datasets, generally from flat and homogenous terrain locations. This leads to high uncertainty of model-based estimates of the above-mentioned variables over steep and complex topography.

Similarly, climate change scenarios fundamentally depend on the assumption that the accuracy of climate models, and in particular of physical process parameterizations, does not change over the temporal horizon of the projection. Evidently, this cannot be taken for granted if parameterizations are subjected to extensive tuning towards present-day climate, potentially leading to simulations plagued by unclear error compensation effects.

These considerations explain why one of the major aims of TEAMx is to contribute to making weather forecasts and climate simulations right for the right reason (that is, based on physically sound principles, subjected to minimal tuning and free of error compensations). Improving the understanding and the representation of atmospheric processes over complex orography is an important part of this general overarching aim.

\subsubsection{Useful observational data}

Field observations will be an integral part of TEAMx to answer the scientific questions outlined in Sec. 2.1-2.3. Data collected from a spatially dense and multi-scale network of diverse observational platforms will provide a basis for improved understanding of the relevant processes and for evaluation of modelling systems and existing model parameterizations, including their underlying assumptions. New theories for parameterizations and new benchmark simulations for MoBL-specific processes can be developed based on this observational dataset.

The combination of high-quality observations, numerical experiments and new theoretical models may help identify needs for the future establishment of additional routine measurements, e.g., for improving error statistics in the assimilation of surface data or for improving the estimates of MoBL depth used in pollutant dispersion modelling. The resulting recommendations can then be implemented by national weather services and environmental agencies.

Finally, testing new remote-sensing techniques against more traditional observations can lead to improvements in their use over complex terrain and thus to the extension of current measurement capabilities in terms of spatial/temporal resolution and coverage.

\subsubsection{Better models}

Nowadays, almost all the weather forecasts conveyed to the general public are based on some kind of modelling product. Through improvements in parameterization schemes based on special observations, TEAMx will contribute to providing more accurate weather and climate information at the local scale in complex terrain, resulting in generally better numerical forecasts and more timely weather warnings. 
Weather and climate information is relevant not only to forecasting, but also to many scientific disciplines and weather-sensitive sectors of the economy. Air pollution studies were dealt with in Sec. 2.1.8, but accurate knowledge of atmospheric properties strongly affected by exchange processes (not only temperature, wind and precipitation) is essential in a number of other disciplines:

(i) Forecasting extreme weather (deep convection, hail and flooding) and weather-related natural hazards (e.g., landslides) is of capital importance for civil protection. Complexterrain areas are particularly exposed to such risks, in particular small catchments where flooding can occur over extremely short timescales. In fact, providing precipitation fields for hydrological run-off modelling is one of the most crucial meteorological services.

(ii) Forecasting the distribution, density, albedo and evolution of the snowpack in mountainous areas, including its redistribution by the wind and its sublimation, is critically important for avalanche warning and, on longer time scales, for water resource management.

(iii) Wildfires, whose propagation depends primarily on fuel availability, can be reinforced by specific weather conditions caused by mountain-wave dynamics, e.g., extremely low near-surface humidity and high wind speeds. Fire forecasting is made challenging by the fact that wildfires are affected by the environmental conditions but also modify them, as is made visible by pyrocumulus clouds.

(iv) Several tasks in agriculture (e.g., irrigation, fertilizing, applying pesticides, operating frost protection systems) can be optimized if good forecasts of the surface weather are available. Information about winds, temperature and moisture is also critical in dealing with public health issues like pollen dispersion or disease spreading.

(v) Some aspects of urban planning, for instance ensuring nocturnal cooling and ventilation of the urban atmosphere, can benefit from the knowledge of local breeze circulations induced by the orography or by urban/rural land-use contrasts.

(vi) The extrapolation of measured wind energy potential to nearby sites is generally based on flat-terrain paradigms, which have numerous limitations over complex orography. Optimal micro-siting often has to deal with meteorological considerations related to near-surface momentum exchange (e.g., orographically-induced wind speed-up and turbulence). Surface exchange of heat and moisture (including snowmelt, evapotranspiration and cloud formation) affects other renewable energy industries too (e.g., hydropower, solar energy).

(vii) Transportation (primarily air traffic, but ground transportation as well) is affected by atmospheric conditions, in particular by visibility, icing, wind and turbulence. Some of the most adverse conditions for traffic in mountainous areas (mountain-wave turbulence, downslope windstorms, fog, low visibility, road-surface freezing) are intimately connected with one or another form of exchange process. Valleys, where major transportation routes are typically confined, are particularly exposed to traffic-related air pollution. Partial relief might result from regulatory systems adopting measures (e.g., speed limits or bans of certain vehicle types) depending on traffic count data and on forecasts of boundary layer depth and mixing, before critical concentration thresholds are reached.

$\rightarrow$ Impact-oriented forecasts must target a broad range of space and time scales. This requires using different tools, from general circulation models to large-eddy simulation codes. Regardless of model resolution, virtually all the weather- and climate model 
output directly relevant to human activities is critically affected by the accuracy of a few key parameterization schemes, which are known to suffer from strong limitations over complex orography (e.g., radiation, turbulence, land-atmosphere exchange, etc.).

$\rightarrow$ Reducing the uncertainty of observations and forecasts to the benefit of end-users demands acquiring application-oriented observational data over orographically complex regions. Since the weather parameters of interest, the optimal observation strategies, the most relevant forecast ranges and the admissible observation and forecast uncertainties are all application-dependent, involvement of weather service users in the planning of any dedicated observational effort is essential.

\section{Relevant literature}

$\rightarrow$ De Wekker, S.F.J., Kossmann, M., Knievel, J.C., Giovannini, L., Gutmann, E.D., Zardi, D. (2018): Meteorological Applications Benefiting from an Improved Understanding of Atmospheric Exchange Processes over Mountains. Atmosphere, 9, 371. 


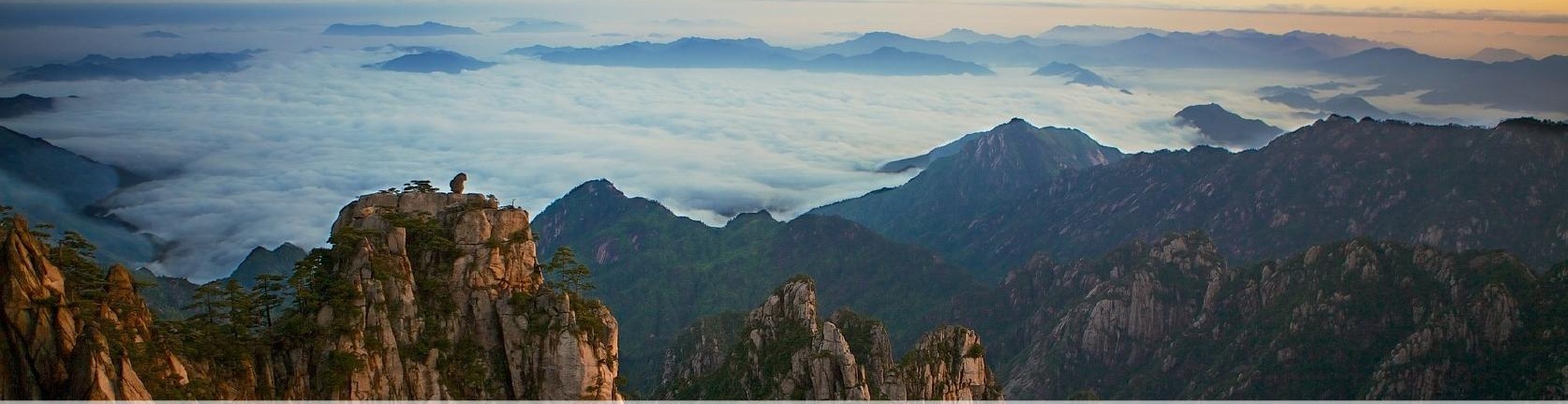

\section{Implementation}

\subsection{Organization}

TEAMx activities are spearheaded by a Coordination and Implementation Group (CIG) established in September 2017 and consisting of 13 members. A Memorandum of Understanding (MoU) has been signed among the institutions that support TEAMx, which initially coincide with the home institutions of the CIG members ("founding partners"). The TEAMx partnership welcomes new members ("additional partners").

In August 2018 a TEAMx Programme Coordination Office (PCO) was created at the University of Innsbruck. As of fall 2019, the TEAMx PCO receives financial support from: Center for Climate Systems Modeling, Karlsruhe Institute of Technology, Meteo France, MeteoSwiss, National Center for Atmospheric Science, University of Innsbruck, University of Trento, and ZAMG.

Basic funding for TEAMx research is expected to come primarily from a bottom-up approach, by which each investigator contributes his/her own resources to the programme, through projects supported by national and international research funding agencies after successful peer-review. A mechanism of "TEAMx endorsement" through support letters written by the TEAMx PCO on behalf of the CIG is in place. This is intended as a way of strengthening research proposals that fit in the overall scope of the programme, by showing that the PIs have a specific role in and work in close connection with a large research community.

In the future, collaborative research within TEAMx will be organized in Scientific Working Groups and coordinated by a Scientific Steering Committee where all working groups are represented. The envisioned phases of TEAMx and their indicative timeline are represented in the following diagram.

\begin{tabular}{|c|c|c|c|c|c|c|c|}
\hline \multirow{2}{*}{ Definition of working groups } & 2019 & 2020 & 2021 & 2022 & 2023 & 2024 & $2025 \ldots$ \\
\hline & & & & & & & \\
\hline Numerical experimentation & & & & & & & \\
\hline Observation phase & & & & & & & \\
\hline
\end{tabular}




\subsection{Observations}

A major goal of TEAMx is to conduct co-ordinated experimental activities in order to: i) Characterize the structure and spatial/temporal variability of the MoBL; ii) Better understand and hence model the relevant exchange processes; iii) Verify current NWP models and validate or train post-processing and downscaling algorithms for weather and climate simulation in mountainous areas.

The TEAMx field phase is tentatively planned for 2023-2024. During the observation period (approximately one year), special instrumentation (flux towers, profilers, cloud radars, airborne in situ and remote sensors) will be deployed in the vicinity of existing long-term micrometeorological observatories in the Alps, complementing and locally enhancing the existing measurement network. The specific challenges of the TEAMx field experiment will be:

$\rightarrow$ High observation density. The processes that contribute to the exchange between the mountain surface and the free troposphere have a high degree of spatial variability. Sampling this variability requires a large number of available instruments, which can only be achieved through cooperation between different groups.

$\rightarrow$ Common procedures for data processing. Having different groups active in the same target area makes it necessary to coordinate the measurement strategies, retrieval algorithms, post-processing options, etc.

$\rightarrow$ Extended temporal coverage. The typical duration of field experiments (up to a few months) is not appropriate for climatological studies and poses interpretation challenges even for meteorological investigations. In particular, the interaction of processes at different spatial scales (e.g., convection initiation occurring in a specific configuration of synoptic flow and thermally driven circulation) makes it unlikely to encounter similar conditions during a sufficient number of days within a single campaign. Existing long-term observational platforms are therefore important for assessing the representativeness or generality of the intensive observations made on individual days, as well as bridging the gap towards climatological time scales.

$\rightarrow$ Concentration of measurement efforts. Different exchange processes may be better observed at different sites. For instance, mountainous regions next to high-emission areas are intuitively better suited for pollutant transport studies. Study of orographic convection might instead require a target area in the vicinity of a climatological maximum of convective rainfall. However, logistical aspects and the necessity of overall scientific coordination require concentration of resources in a limited number of sites.

Although TEAMx will maintain a strong focus on the Alps, it is envisaged that partnerships with other regionally focused initiatives may lead to joint or coordinated experimental strategies, or even to an expansion of TEAMx to other regions. This might prove necessary because the climate of the European Alps is not representative of that of mountains worldwide. The Alps are extensively covered by vegetation and receive precipitation more or less continuously throughout the year. Other major mountain ranges worldwide, like the Andes or the Himalayas, are under very different climatic conditions in the subtropics and generally mark a sharp transition between relatively moist and very arid regions. Given the marked impact of soil and vegetation on exchange processes, observational efforts in climate areas other than the mid-latitudes might prove necessary, in coordination with TEAMx.

For extensive discussion of the essential instruments, target areas, special observation periods and long-term monitoring, see the TEAMx Experimental Plan (currently in preparation). 


\subsection{Numerical experimentation}

A recent survey of systematic errors in weather and climate models (WMO Working Group on Numerical Experimentation, October 2018), receiving 35 answers from 14 different modelling centres, defined a ranking of the model errors that need to be addressed with the highest priority. Processes tightly related to surface exchange are on top of the list, and include convective precipitation, surface fluxes, diurnal cycles. Albeit with lower priority, orographic precipitation and surface drag found their place in the ranking too.

A coherent plan to identify and possibly reduce systematic model errors over mountainous terrain should be based both on idealized, process-based numerical experimentation, and on the routine verification of operational NWP.

$\rightarrow$ Idealized numerical experiments. Idealized simulation has been widely exploited to explore the mechanisms of a variety of MoBL exchange processes, normally using drastic simplifications in initial states and orography profiles. Future research should work towards reducing the level of idealization by incorporating at least some elements of complexity, which are known to greatly affect the intensity of exchange in the real world (e.g., background flow variability, small-scale terrain irregularities, landcover heterogeneity, spatially- and temporally-variable solar forcing). Collaborative research based on idealized simulations may serve in particular the purposes of model evaluation and parameterization development.

$\rightarrow$ Observation-based benchmarks. While observational data have typically not been a concern in intercomparison studies dealing with mesoscale phenomena, they have been central to those addressing boundary-layer and surface-layer processes, for instance in the GEWEX Atmospheric Boundary Layer Study (GABLS). All GABLS studies to date considered flat and homogenous terrain. Complex-terrain benchmark cases with an overarching logic similar to GABLS are a useful way of making progress in complex-terrain modelling. They will need to: i) Address separately the role of orographic and land-cover complexity; ii) Include different types of model grids and treatment of the surface, including immersed and embedded boundary methods; iii) Replace single-column models by small-sized regional domains, in order to retain a level of terrain complexity and explore the problems posed by horizontal exchange parameterization.

$\rightarrow$ Performance of operational NWP models over complex terrain. The conjecture that numerical weather and climate simulations are plagued by larger systematic errors near mountains than over flat terrain has not been solidly proven so far. Systematic comparison of model performance over flat and complex orography is out of the scope of routine forecast verification, and can become the subject of renewed collaboration in LAM verification. A complex-terrain verification study should consider a temporal range of a few years and a set of measurement stations at morphologically different sites, covering a major mountain range. Also, it should not be limited to precipitation, temperature and wind, but also consider quantities related to surface exchange (e.g., surface energy budget components, low-level cloud fraction, mixing heights). Such a study could be further extended by evaluating the skill of the best available models, i.e., turbulence-resolving simulations (LES) of selected case studies, initialized by assimilating special field observations.

Since the meteorological services of the Alpine countries are all either active or prospective partners of TEAMx, during the programme it will be possible to access to operational nu- 
merical weather prediction output and model diagnostics from several limited-area modelling consortia in Europe (COSMO, ALADIN, LACE, Met Office). On the climate modelling side, a CORDEX Flagship Pilot Study on convection-permitting climate simulation in the Alpine region is being planned within TEAMx. A detailed modelling strategy based on process-based idealized simulations and a verification study will be included in the TEAMx Experimental Plan. 


\section{Acronyms}

ABL

ALADIN

ALPEX

BVOC

CI

CIG

COPS

CORDEX

COSMO

DA

EDW

GABLS

GEWEX

IPCC

LACE

LAM

LES

LPDM

MAP

MoBL

$\mathrm{MoU}$

NWP

PYREX

RANS

RPAS

SBL

SGS

SOA

TEAMx

UAV

VOC

WCRP

WMO
Atmospheric Boundary Layer

Aire Limitée Adaptation dynamique Développement InterNational

Alpine Experiment

Biogenic Volatile Organic Compounds

Convection Initiation

Coordination and Implementation Group

Convective and Orographically-induced Precipitation Study

COordinated Regional Downscaling EXperiment

Consortium for Small-scale Modeling

Data Assimilation

Elevation Dependent Warming

GEWEX Atmospheric Boundary Layer Study

Global Energy and Water Exchanges

Intergovernmental Panel on Climate Change

Limited Area modelling in Central Europe

Limited-Area Model

Large-Eddy Simulation

Lagrangian Particle Dispersion Model

Mesoscale Alpine Programme

Mountain Boundary Layer

Memorandum of Understanding

Numerical Weather Prediction

Pyrénées Experiment

Reynolds-Averaged Navier-Stokes equations

Remotely Piloted Aircraft Systems

Stable Boundary Layer

Sub-Grid-Scale

Secondary Organic Aerosol

Multi-scale Transport and Exchange Processes in the Atmosphere over Mountains -

Programme and eXperiment

Unmanned Aerial Vehicles

Volatile Organic Compounds

World Climate Research Programme

World Meteorological Organization 
Document structure based on The Legrand Orange Book

LATEX template downloaded from http://www . LaTeXTemplates . com

\section{Image credits}

Page 5: Public domain.

https://pixabay.com/photos/mountains-sunrise-dawn-beautiful-1985027/

Page 9: NASA Worldview, Terra/MODIS corrected reflectance imagery, 15 November 2018. https://earthdata.nasa.gov/labs/worldview/

Page 13: Valle Riviera, Switzerland, 1999. Courtesy of Andreas Weigel.

http: //www . teamx-programme .org/slider_pictures/1/Andreas-riviera.jpg

Page 37: Public domain.

https://pixabay.com/photos/yellow-mountains-mountain-landscape-532857/ 
TEAMx (www.teamx-programme.org) is an international research programme that aims at improving the understanding of exchange processes in the atmosphere over mountains at multiple scales and at advancing the parameterizations of these processes in numerical models for weather and climate prediction. This document, compiled by the TEAMx Programme Coordination Office, provides a concise overview of the scientific scope of TEAMx. 